inhibition selectively sensitizes G1 checkpoint-deficient cells to lethal premature chromatin condensation. Proc Natl Acad Sci U S A. 2001;98(16):9092-9097.

8. Koniaras K, Cuddihy AR, Christopoulos H, Hogg A, O'Connell MJ. Inhibition of Chk1-dependent G2 DNA damage checkpoint radiosensitizes p 53 mutant human cells. Oncogene. 2001;20(51):7453-7463.

9. Zhou BB, Bartek J. Targeting the checkpoint kinases: chemosensitization versus chemoprotection. Nat Rev Cancer. 2004;4(3):216-225.

10. Ma CX, Janetka JW, Piwnica-Worms H. Death by releasing the breaks: CHK1 inhibitors as cancer therapeutics. Trends Mol Med. 2011;17(2):88-96

11. Fracasso PM, et al. A Phase 1 study of UCN-01 in combination with irinotecan in patients with resistant solid tumor malignancies. Cancer Chemother Pharmacol. 2010;67(6):1225-1237.

12. Reinhardt HC, Aslanian AS, Lees JA, Yaffe MB. p53deficient cells rely on ATM- and ATR-mediated checkpoint signaling through the p38MAPK/MK2 pathway for survival after DNA damage. Cancer Cell. 2007;11(2):175-189.

13. Gilad O, et al. Combining ATR suppression with oncogenic Ras synergistically increases genomic instability, causing synthetic lethality or tumorigenesis in a dosage-dependent manner. Cancer Res. 2010;70(23):9693-9702

14. Toledo LI, et al. A cell-based screen identifies ATR inhibitors with synthetic lethal properties for cancer-associated mutations. Nat Struct Mol Biol. 2011;18(6):721-727.

15. Ferrao PT, Bukczynska EP, Johnstone RW, McArthur GA. Efficacy of CHK inhibitors as single agents in MYC-driven lymphoma cells. Oncogene. 2012;31(13):1661-1672

16. Murga M, et al. Exploiting oncogene-induced replicative stress for the selective killing of Myc-driven tumors. Nat Struct Mol Biol. 2011;18(12):1331-1335.
17. Schoppy DW, et al. Oncogenic stress sensitizes murine cancers to hypomorphic suppression of ATR. J Clin Invest. 2012;122(1):241-252.

18. Bartkova J, et al. DNA damage response as a candidate anti-cancer barrier in early human tumorigenesis. Nature. 2005;434(7035):864-870.

19. Gorgoulis VG, et al. Activation of the DNA damage checkpoint and genomic instability in human precancerous lesions. Nature. 2005;434(7035):907-913.

20. Brown EJ, Baltimore D. ATR disruption leads to chromosomal fragmentation and early embryonic lethality. Genes Dev. 2000;14(4):397-402.

21. Ruzankina Y, et al. Deletion of the developmentally essential gene ATR in adult mice leads to agerelated phenotypes and stem cell loss. Cell Stem Cell. 2007;1(1):113-126

22. Reaper PM, et al. Selective killing of ATM- or p53deficient cancer cells through inhibition of ATR. Nat Chem Biol. 2011;7(7):428-430.

\title{
Hemolysis and cell-free hemoglobin drive an intrinsic mechanism for human disease
}

\author{
Mark T. Gladwin, ${ }^{1}$ Tamir Kanias, ${ }^{1}$ and Daniel B. Kim-Shapiro ${ }^{2}$
}

${ }^{1}$ Vascular Medicine Institute and Pulmonary, Allergy and Critical Care Medicine, Department of Medicine, University of Pittsburgh, Pittsburgh, Pennsylvania, USA. ${ }^{2}$ Department of Physics and Translational Science Center, Wake Forest University, Winston-Salem, North Carolina, USA.

\begin{abstract}
Blood transfusion represents the first and most prescribed cell-based therapy; however, clinical safety and efficacy trials are lacking. Clinical cohort studies have suggested that massive transfusion and/or transfusion of aged stored blood may contribute to multiorgan dysfunction in susceptible patients. In this issue of the JCI, Baek and colleagues report that aged stored blood hemolyzes after massive transfusion in a guinea pig model. Hemolysis led to vascular and kidney injury that was mediated by cell-free plasma hemoglobin and prevented by coinfusion of the specific hemoglobin scavenger protein, haptoglobin. These studies support an expanding body of research indicating that intravascular hemolysis is a pathological mechanism in several human diseases, including multiorgan dysfunction after either massive red blood cell transfusion or hemoglobin-based blood substitute therapy, the hemoglobinopathies, malaria, and other acquired and genetic hemolytic conditions.
\end{abstract}

\section{Blood transfusion and the storage lesion}

Blood transfusion is one of the first and most prescribed cell-based therapies. Despite the frequency with which blood transfusion is prescribed, the timing, dose, and established placebo-adjusted benefits of this "drug" have not been established. Blood transfusion is clearly beneficial in a multitude of clinical conditions, such as massive traumatic and surgical hemorrhage, critical anemia, and anemia associated with

Conflict of interest: Mark T. Gladwin and Daniel B. Kim-Shapiro are named as co-inventors on an NIH government patent for the use of nitrite salts for cardiovascular conditions.

Citation for this article: J Clin Invest. 2012; 122(4):1205-1208. doi:10.1172/JCI62972. ischemic heart disease; it is also clearly beneficial as a supportive and exchange therapy for hemoglobinopathies. However, an increasing number of studies suggest that massive transfusion - defined as the transfusion of approximately one complete blood volume within the first 24 hours of resuscitation - may increase the risk of multiorgan dysfunction, respiratory and renal insufficiency, and death (1-5).

Studies in patients who have undergone cardiac surgery or experienced trauma or critical illness have suggested that the age of the transfused blood may relate to the risk of adverse clinical outcomes (1). The combined effects of storage on red blood cells have been termed the "red blood cell storage lesion." These effects modulate membrane integrity (e.g., reducing deformability and increasing rigidity), alter rheological properties, and cause hemolysis and have been proposed to contribute in some way to the risk of transfusion of aged blood. However, the epidemiologically established relationship between age of transfused blood and risk of adverse clinical outcomes is confounded by a number of important variables. First, transfusion of multiple units of blood increases the probability that an older unit is given, creating uncertainty about the role of massive transfusion as opposed to that from a storage lesion mechanism (2). Second, sicker patients receive more units (3). Finally, O blood group units are more rare and are more rapidly depleted from the inventory, so that patients who have type $\mathrm{O}$ blood are more likely to receive fresh blood, leading to fundamental differences among groups in published case-controlled cohort studies that may influence outcomes (4).

In order to address the major variables confounding the assessment of risk of transfusing blood stored for a long time, NIH-funded well-controlled transfusion studies in preclinical animal models and human placebo-controlled clinical trials are being performed. While the clinical trials are underway, because of safety and ethical considerations, these trials evaluate only modest ranges of storage time as 


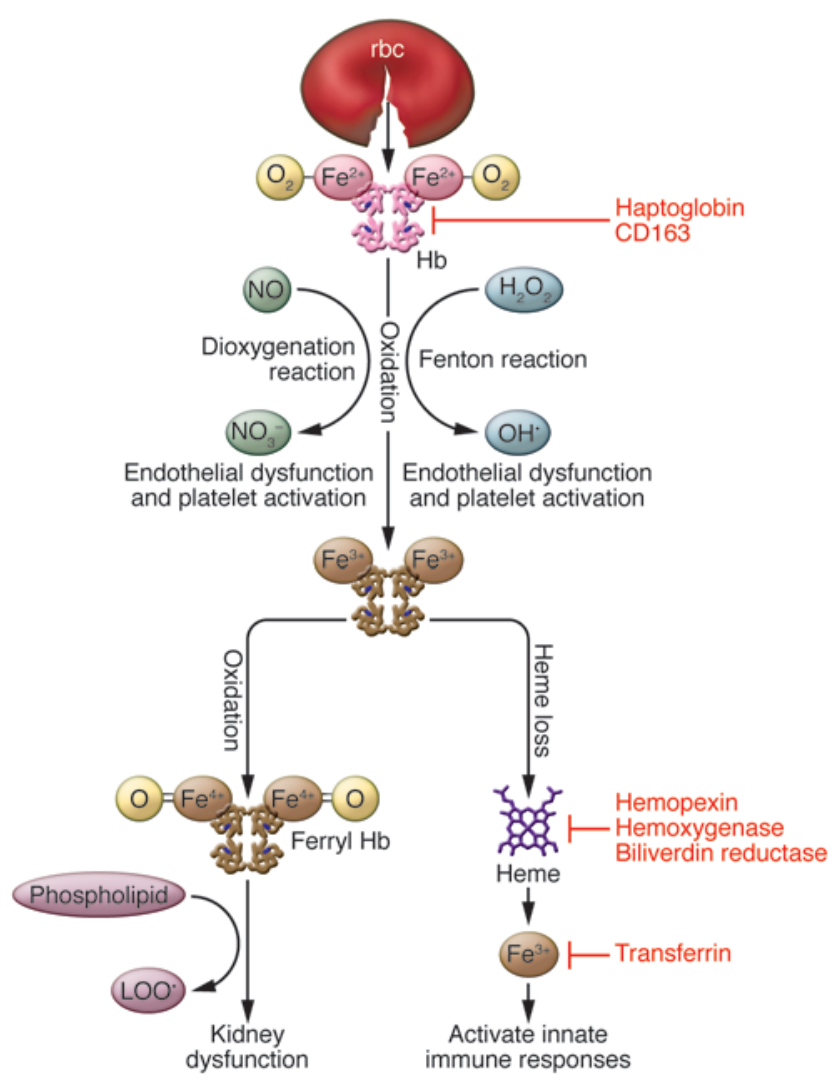

\section{Figure 1}

Mechanisms by which hemolysis and cell-free hemoglobin can mediate vascular injury. Intravascular hemolysis releases cell-free hemoglobin $(\mathrm{Hb})$ into the plasma. The hemoglobin is maintained in the ferrous redox state, which will react with NO in a near-diffusion limited reaction to inhibit NO signaling. Extravasation of hemoglobin in the subendothelial space and reductive recycling back to the ferrous state may enhance this pathological effect. Oxidation of hemoglobin, particularly in the kidney, from the ferric state to the ferryl state may further drive fenton and peroxidase oxidative cellular injury (involving the lipid peroxyl species, LOO•), leading to acute kidney injury. Free heme and iron promote inflammatory injury via activation of innate immune responses in macrophages and monocytes. Compensatory pathways that normally control these NO dioxygenation and oxidation reactions include haptoglobin- and hemopexin-mediated sequestration of hemoglobin dimer and heme, respectively. Downstream Nrf-2, hemoxygenase, and biliverdin reductase signaling detoxify heme and iron and provide catalytic antioxidant, antiproliferative, and antiinflammatory protective signaling. well as limited transfusion volumes and patients with lower severity of illness. It is possible that a deleterious effect of red cell storage may only be observed with the longest storage time, with massive transfusion, and/or in susceptible recipients. In contrast, preclinical animal model studies uniformly evaluate massive transfusion at the extremes of storage duration.

In this issue of the JCI, Baek and colleagues evaluate a preclinical animal model of massive transfusion (5). They report the effects in guinea pigs of massive transfusion of either fresh blood stored for 2 days or blood stored for 21 or 28 days. They found that the older blood caused hypertension, vascular injury, and renal insufficiency and that this was directly related to the intensity of posttransfusion intravascular hemolysis and the levels of cell-free, nonhaptoglobin-bound, plasma hemoglobin. These findings are consistent with recent observations that blood transfusion can mediate endothelial injury and impaired vascular function as a result of the most fundamental of storage lesions, hemolysis (6-8). The beneficial effects they observe with coinfusion of haptoglobin (5) confirm a mechanism intrinsic to hemoglobin and identify a new potential therapy.

\section{Intravascular hemolysis and the storage lesion}

Using red cell ektacytometry and shearstress analysis, Baek and colleagues report that red blood cell membrane rigidity increased at differing rates for human and guinea pig red blood cells as a function of storage duration (5), with human blood stored for 28 days comparable to guinea pig blood stored for 14 days and human blood stored for 42 days (which is the limit of storage in the US) comparable to guinea pig blood stored for 28 days. After massive exchange transfusion of $80 \%$ of the blood volume with leukocyte-depleted stored blood, substantial intravascular hemolysis was observed, together with accumulation of reduced ferrous oxyhemoglobin in the plasma of animals receiving old blood. While the transfusion of new blood was not associated with changes in blood pressure, old blood was observed to have marked acute hypertensive effects, which were coupled to increases in the NO-consuming properties of plasma and in vivo reduction of levels of NO metabolites (nitrate and nitrite). Interestingly, old blood but not new blood caused coagulative necrosis of the aortic arch, from the luminal tunica intima to the tunica media, and this was associated with iron deposition within the supporting vasa vasorum. Major effects on renal function were also observed, with upregulation of $\mathrm{Nrf}-2$-dependent antioxidant gene expression signatures; dilation of proximal and distal tubules, which is consistent with tubular degeneration; and elevation of serum levels of creatinine. Importantly, washing the stored red blood cells did not prevent these effects, as the aged cells continued to hemolyze intravascularly after transfusion.

Given the association between the negative effects of transfusion of old blood and substantial intravascular hemolysis, resulting in accumulation of reduced ferrous oxyhemoglobin, Baek and colleagues infused the high-affinity hemoglobin scavenger haptoglobin along with the stored blood (5). Upon binding to hemoglobin, haptoglobin is cleared from the circulation via the monocyte/macrophage haptoglobin-hemoglobin scavenger receptor CD163. Based on observations that the vasoactive effects, vascular injury, and renal Nrf-2-dependent gene expression changes and dysfunction were ameliorated by coinfusion of haptoglobin with the aged blood, the authors propose that the pathophysiology of transfusion of aged 
stored blood is intrinsically mediated by cell-free plasma hemoglobin. These findings are consistent with recent observations that the storage lesion may relate, at least in part, to the fundamental effects of hemolysis and cell-free plasma hemoglobin on vascular function (6-8).

\section{Rust reactions - pleiotropic toxicity of cell-free plasma hemoglobin, heme, iron, and oxygen}

The new studies of red blood cell storage (5-7) support a growing appreciation that intravascular hemolysis represents a fundamental mechanism for human disease (9-11). This concept evolved from clinical trial observations that trauma patients receiving hemoglobin-based oxygen carriers developed hypertension and multiorgan injury caused by hemoglobin-NO scavenging reactions (12-14) and clinical observations of vasculopathic complications in patients with hemoglobinopathies and hemolytic anemia $(15,16)$. Intravascular hemolysis releases hemoglobin and enzymes, such as arginase 1, into plasma. An efficient hemoglobin and heme scavenging system, consistenting of haptoglobin, CD163, and hemopexin, sequesters these redox-active molecules. When these scavenging systems are saturated by excess hemoglobin, cell free plasma hemoglobin accumulates and extravasates within the vasculature and organs (10).

A number of NO depletion and ROS generation pathological pathways mediated by cell-free plasma hemoglobin have been defined (Figure 1). A primary effector is oxyhemoglobin, which is largely maintained in the reduced state in plasma and can react in a rapid and irreversible dioxygenation reaction with $\mathrm{NO}$ to form methemoglobin and nitrate $(7,17)$. This reaction of NO with oxyhemoglobin accounts for the acute vasoactive responses seen with infusions of hemoglobin-based oxygen carriers and cell-free plasma hemoglobin (14). It also causes a NO resistance state of endothelial dysfunction in diseases such as sickle cell and malaria $(9,18,19)$. The hypertensive effects of cell-free plasma hemoglobin relate to the disruption of important NO diffusional barriers between the endothelium and the normally compartmentalized intracellular hemoglobin. NO normally is able to signal because the hemoglobin reactions are effectively shielded by a cell-free zone between the endothelium and red blood cells caused by laminar flow and the glycocalyx, by an unstirred layer around the red blood cell, and by an intrinsic NO diffusional barrier in the red blood cell submembrane (20). Accordingly, both the red blood cell and haptoglobin prevent hemoglobin extravasation into the subendothelial compartments in which $\mathrm{NO}$ scavenging can directly interrupt NO diffusion to smooth muscle.

The toxicity of intravascular cell-free hemoglobin does not stop at NO scavenging. Additional oxidation reactions are catalyzed by hemoglobin, heme, and iron. Reactions of hydrogen peroxide with ferrous and ferric hemoglobin drive the fenton and peroxidase reactions to generate hydroxyl and ferryl heme radicals. These oxidation reactions can redox cycle, leading to lipid peroxidation and cellular injury. Recent studies have highlighted the importance of this reaction in the renal injury that is associated with myoglobinemia from rhabdomyolysis (21), which appears pathologically similar to the kidney injury observed by Baek and colleagues (5). Downstream reactions may relate to heme and iron release from the hemoglobin protein. Heme can amplify innate immune responses in sepsis (22), while free iron can amplify inflammatory responses after the intracellular uptake and catabolism of a subset of damaged, stored red blood cells by the monocyte/ macrophage system (23). These studies, in sum, highlight the remarkable toxicity of hemoglobin, iron, heme, and oxygen when liberated from the reductive intracellular red blood cell environment.

\section{Hemoglobin complex size and extravasation matter}

An interesting and yet to be explained observation of Baek and colleagues is that haptoglobin binding to hemoglobin reduces the vasoconstrictive effects of hemoglobin, yet paradoxically increases the half-life of hemoglobin in the circulation and does not inhibit the intrinsic reactivity of hemoglobin with NO (5). While the mechanism(s) for this was not explored, the observations are consistent with extravasation of hemoglobin and subcellular compartmental NO inhibition. The mixtures of haptoglobin used by Baek and colleagues include all three of the haptoglobin isoforms (1-1, $1-2$, and 2-2), which form disulfide bonds and high-molecular weight multimers. The vasopressor effect of hemoglobin-based oxygen carriers has been shown to correlate with $\mathrm{NO}$ scavenging reaction rates and with molecular size $(14,17)$; the higher the molecular size of the hemoglobin, the lesser the hypertensive effect. It has been proposed that the large molecular mass limits extravasation. It is therefore likely that high mass hemoglobin-haptoglobin multimers will not extravasate and will exhibit reduced NO scavenging, and this is consistent with the reduced renal clearance of free hemoglobin observed by Baek and colleagues after haptoglobin infusion (5). Future studies to image labeled hemoglobin extravasation coupled to studies of in vivo endothelial NO signaling will be necessary to test the effects of haptoglobin on hemoglobin extravasation and NO inhibition in the subendothelial compartment.

\section{Conclusion - potentially novel therapy to mitigate hemolysis- mediated vasculopathy}

The work of Baek and colleagues, in this issue of the JCI (5), supports the hypothesis that intravascular hemolysis represents a fundamental mechanism for human disease $(9,10)$. The hemoglobin-mediated vasculopathy and end-organ toxicity is likely relevant to hemolysis in malaria, cardiopulmonary bypass, and genetic red cell enzymopathies, membranopathies, and hemoglobinopathies. A potent role for haptoglobin in limiting end-organ injury opens the door to new therapies targeting hemoglobin and heme sequestration and catabolism. Infusions of haptoglobin and hemopexin have now clearly been shown to limit hemoglobinand heme-mediated toxicity in preclinical models of blood transfusion and sepsis (22). These findings raise interesting questions surrounding potential protective effects of plasma or whole blood transfusion to increase the concentration of haptoglobin and hemopexin and provide a rationale for the development of haptoglobin-enriched and hemopexin-enriched blood products or recombinant protein preparations. While the high-molecular weight of these molecules provides challenges to recombinant protein generation and delivery, alternative approaches using aptamer and small-molecule technology may be successful. The design and testing of hemoglobin-binding and hemoglobin-metabolism therapeutics should be considered for transfusion of stored blood, cardiopulmonary bypass, malaria, and hemoglobinopathies.

\section{Acknowledgments}

M.T. Gladwin receives research support from NIH grants R01HL098032, RO1HL096973, and PO1HL103455; the 
Institute for Transfusion Medicine; and the Hemophilia Center of Western Pennsylvania.

Address correspondence to: Mark T. Gladwin, Pulmonary, Allergy and Critical Care Medicine, University of Pittsburgh, Pittsburgh, Pennsylvania 15261, USA. Phone: 412.692.2210; Fax: 412.692.2260; E-mail: gladwinmt@upmc.edu.

1. Wang D, Sun J, Solomon SB, Klein HG, Natanson C. Transfusion of older stored blood and risk of death: a meta-analysis [published online ahead of print December 21, 2011]. Transfusion. doi:10.1111/ j.1537-2995.2011.03466.x.

2. van de Watering L, Lorinser J, Versteegh M, Westendord R, Brand A. Effects of storage time of red blood cell transfusions on the prognosis of coronary artery bypass graft patients. Transfusion. 2006; 46(10):1712-1718

3. Corwin HL, et al. The CRIT Study: Anemia and blood transfusion in the critically ill-current clinical practice in the United States. Crit Care Med. 2004;32(1):39-52.

4. Koch CG, et al. Duration of red-cell storage and complications after cardiac surgery. N Engl J Med. 2008; 358(12):1229-1239.

5. Baek JH, et al. Hemoglobin-driven pathophysiology is an in vivo consequence of the red blood cell storage lesion that can be attenuated in guinea pigs by haptoglobin therapy. J Clin Invest. 2012; 122(4):1444-1458.

6. Kim-Shapiro DB, Lee J, Gladwin MT. Storage lesion: role of red blood cell breakdown. Transfusion. 2011;51(4):844-851.

7. Donadee $C$, et al. Nitric oxide scavenging by red blood cell microparticles and cell-free hemoglobin as a mechanism for the red cell storage lesion. Circulation. 2011;124(4):465-476.

8. Lee JS, Gladwin MT. Bad blood: the risks of red cell storage. Nat Med. 2010;16(4):381-382.

9. Reiter CD, et al. Cell-free hemoglobin limits nitric oxide bioavailability in sickle-cell disease. Nat Med. 2002;8(12):1383-1389.

10. Rother RP, Bell L, Hillmen P, Gladwin MT. The clinical sequelae of intravascular hemolysis and extracellular plasma hemoglobin: a novel mechanism of human disease. JAMA. 2005;293(13):1653-1662.

11. Minneci PC, et al. Hemolysis-associated endothelial dysfunction mediated by accelerated NO inactivation by decompartmentalized oxyhemoglobin. J Clin Invest. 2005;115(12):3409-3417.

12. Sloan EP, et al. Diaspirin cross-linked hemoglobin (DCLHb) in the treatment of severe traumatic hemorrhagic shock: a randomized controlled efficacy trial. JAMA. 1999;282(19):1857-1864.

13. Natanson C, Kern SJ, Lurie P, Banks SM, Wolfe SM. Cell-free hemoglobin-based blood substitutes and risk of myocardial infarction and death: a metaanalysis. JAMA. 2008;299(19):2304-2312.

14. Olson JS, Foley EW, Rogge C, Tsai AL, Doyle MP, Lemon DD. No scavenging and the hypertensive effect of hemoglobin-based blood substitutes. Free Radic Biol Med. 2004;36(6):685-697.

15. Gladwin MT, et al. Pulmonary hypertension as a risk factor for death in patients with sickle cell disease. NEngl J Med. 2004;350(9):886-895.

16. Aessopos A, et al. Cardiac involvement in thalassemia intermedia: a multicenter study. Blood. 2001; 97(11):3411-3416.

17. Doherty DH, et al. Rate of reaction with nitric oxide determines the hypertensive effect of cell-free hemoglobin. Nat Biotechnol. 1998;16(7):672-676.

18. Yeo TW, et al. Relationship of cell-free hemoglobin to impaired endothelial nitric oxide bioavailability and perfusion in severe falciparum malaria. J Infect Dis. 2009;200(10):1522-1529.

19. Janka JJ, et al. Increased pulmonary pressures and myocardial wall stress in children with severe malaria. J Infect Dis. 2010;202(5):791-800.

20. Kim-Shapiro DB, Schechter AN, Gladwin MT. Unraveling the reactions of nitric oxide, nitrite, and hemoglobin in physiology and therapeutics. Arterioscler Thromb Vasc Biol. 2006;26(4):697-705.

21. Boutaud O, et al. Acetaminophen inhibits hemoprotein-catalyzed lipid peroxidation and attenuates rhabdomyolysis-induced renal failure. Proc Natl Acad Sci U S A. 2010;107(6):2699-2704.

22. Larsen $\mathrm{R}$, et al. A central role for free heme in the pathogenesis of severe sepsis. Sci Transl Med. 2010;2(51):51ra71.

23. Hod EA, et al. Transfusion of red blood cells after prolonged storage produces harmful effects that are mediated by iron and inflammation. Blood. 2010;115(21):4284-4292. 